\title{
O Papel da Orientação Empreendedora e da Informação de Mercado na Geração de Inovação Radical
}

\author{
Marcelo Gattermann Perin \\ Pontifícia Universidade Católica do Rio Grande do Sul - PUCRS \\ mperin@pucrs.br \\ Cláudio Hoffmann Sampaio \\ Pontifícia Universidade Católica do Rio Grande do Sul-PUCRS \\ csampaio@pucrs.br \\ Cláudia Simões \\ Universidade do Minho - Portugal \\ csimoes@eeg.uminho.pt
}

\section{RESUMO}

Apesar do grande interesse e da relativa consolidação teórica a respeito do tema de antecedentes da inovação radical, uma série de estudos vem demonstrando que ainda há recursos organizacionais que podem representar participação importante neste fenômeno organizacional. Visando um aprofundamento deste tema, este trabalho aborda o papel do capital social externo como elemento moderador no impacto de dois antecedentes na criação de inovação radical, especificamente a orientação ao empreendedorismo e a geração de informação. O estudo envolveu empresas brasileiras de diversos setores. Um modelo teórico foi desenvolvido e testado pela aplicação da técnica de modelagem de equações estruturais e análise multigrupo. Os resultados encontrados apontam para uma situação em que a intensificação do capital social externo não registra impacto significativo no caráter empreendedor das empresas quando do desenvolvimento de inovação radical e, surpreendentemente, diminuiu significativamente a participação das informações advindas do mercado neste processo de desenvolvimento.

Palavras-chave: Orientação ao Empreendedorismo, Geração de Informação de Mercado, Inovação.

\begin{abstract}
Despite the great interest and the relative theoretical background about radical innovation, a series of studies has demonstrated that there are still a number of organizational resources that can represent an important role in this organizational phenomenon. This paper addresses the role of external social capital as a moderator on the impact of two antecedents of radical innovation, specifically the entrepreneur orientation and market information generation. The study included Brazilian companies from several sectors. A theoretical model was developed and tested by applying the technique of structural equation modeling and multi-group analysis. The results point to a situation in which the intensification of social capital does not expressed significant change on the entrepreneurial orientation impact on the companies' capability for developing radical innovation and, surprisingly, significantly reduced the importance of market information in this process.
\end{abstract}

Keywords: Entrepreneur Orientation, Market Information Generation, Innovation.

\section{Introdução}

Os recursos e ativos empresariais distintivos e de difícil imitação constituem-se em antecedentes das inovações empresariais de sucesso (BARNEY, 1991; TEECE; PISANO; SHUEN, 1997). Dentre esses recursos, destaca-se o papel da aprendizagem como um relevante condutor para a mitigação dos riscos e para o desenvolvimento de inovação radical (breakthrough innovation) (FLORIN; LUBATKIN; SCHULZE, 2003; HARRISON; BOYLE, 2006; SHANE; VENKATARAMAN 2000; TYAGI 2006), entendida como mudanças tecnológicas significativas.

O ponto de partida do processo de aprendizagem organizacional é a geração de informações de mercado. 
A aquisição da informação pode se originar de experiências diretas, experiências de outros ou mesmo da própria memória organizacional (GARVIN, 1993). Este processo faz uso de instrumentos mais específicos como as pesquisas de mercado, atividades de inteligência competitiva e pesquisas de satisfação de clientes e também de práticas mais amplas como joint ventures e alianças estratégicas (MOORMAN, 1995). Os meios utilizados para a aquisição determinam uma geração de informações mais focadas em experiências internas (exploitation) ou em experiências externas da organização (exploration) (SLATER; NARVER, 1995).

A geração de informação de mercado potencializa a aprendizagem organizacional e esta, por sua vez, pode ser promovida por condições internas e externas à firma. Dentre os fatores internos, três construtos relacionados à cultura empresarial que podem promover a aprendizagem têm recebido especial atenção da literatura de marketing: orientação para o mercado (JAWORSKI; KOHLI 1993), orientação para a aprendizagem (SINKULA; BAKER; NOORDEWEIR 1997) e orientação para o empreendedorismo (LUMPKIN; DESS 1996). Das três orientações, a orientação para o mercado e a orientação para a aprendizagem possuem um corpo de publicação relevante no Brasil, em especial a relação desses construtos com a performance empresarial nas publicações encabeçadas principalmente por Perin e Sampaio (VIEIRA, 2008). Entretanto, justamente $a$ orientação para o empreendedorismo, que tem recebido pouca atenção da literatura de marketing no Brasil, tem sido considerada como a mais intimamente relacionada com a inovação organizacional radical (SLATER; NARVER, 1995). Lumpkin e Dess (1996) definem orientação ao empreendedorismo como os métodos, práticas e estilos de tomadas de decisão que os gestores utilizam para agir de forma empreendedora, incluindo processos de captura de novas oportunidades de produto-mercado e pré-disposição ao risco. A orientação ao empreendedorismo é uma filosofia empresarial que requer um tipo específico de conhecimento organizacional denominado de aprendizagem exploratória (LEVINTHAL; MARCH, 1993). A aprendizagem exploratória contribui para o desenvolvimento de produtos inovadores pelo aumento da diversidade no ingresso de informações na empresa (ROWLEY; BEHRENS; KRACKHARDT, 2000).

Em relação aos fatores externos que promovem a aprendizagem, outro conceito central que tem recebido pouca atenção da literatura de marketing, qual seja, o capital social (COLEMAN, 1988; GRAYSON, 2007; KOKA; PRESCOTT, 2002; YLI-RENKO; AUTIO; SAPIENZA, 2001), denotando a rede de relacionamentos externos e internos de uma unidade social (NAHAPIET; GHOSHAL, 1998). O capital social externo, foco do presente artigo, em função dos relacionamentos externos à empresa, é o que mais provavelmente traz novas informações, permitindo aos gestores não apenas questionar o status quo de sua empresa, mas combinar diversos conhecimentos para celebrar uma maneira única e potencial para novas idéias, novas capacidades e novos produtos, incrementando a capacidade de inovação da empresa (CASEY; ORWALL, 2007; STAM; ELFRING, 2008), o acesso a informação diversificada (ATUAHENE-GIMA; MURRAY, 2007; PERRY-SMITH; SHALLEY, 2003; RINDFLEISCH; MOORMAN, 2001) e mitigando o risco (GIELNES; STEENKAMP, 2007; TYAGI, 2006).

Neste sentido, este artigo objetivou validar um modelo conceitual que busca avaliar o impacto de um conjunto de recursos da empresa, com maior ênfase na orientação ao empreendedorismo e na geração de informação de mercado, procurando descrever o seu impacto na inovação radical, além do efeito moderador do capital social externo nestas relações. A elaboração do modelo teórico teve como base as contribuições prévias na área.

\section{Construção do Modelo Teórico}

Na concepção teórica de Baker, Grinstein e Harmancioglu (2009), consistente com outras formulações recentes (ATUAHENE-GIMA, 1996; HAN et al. 1998; BAKER; SINKULA, 1999, 2005), a orientação ao empreendedorismo é um construto que tem raízes no processo de aprendizagem (BAKER; SINKULA 1999; SLATER; NARVER 1995). Em termos teóricos, a orientação ao empreendedorismo tem sido usualmente associada com três principais dimensões: propensão ao risco, à próatividade e à inovação (COVIN; SLEVIN, 1991). A propensão à inovação refere-se à disposição da empresa para apoiar a criatividade e a experimentação no desenvolvimento de novos produtos, adoção de tecnologia e processos e procedimentos internos, refletindo a prontidão para divergir do status quo e adotar novas ideias (LUMPKIN; DESS, 1996). A pró-atividade refere-se à habilidade da empresa em aproveitar as oportunidades do ambiente e à habilidade de agir em antecipação a futuras mudanças no mercado (CONANT; MOKWA; VARADARAJAN, 1990). A propensão ao risco diz respeito à disposição dos proprietários ou dos gestores em comprometer relevantes recursos empresariais a novos projetos e a nova busca de oportunidades (LUMPKIN; DESS, 1996). A orientação ao empreendedorismo, na verdade, permite que a empresa esteja receptiva às oportunidades emergentes e, portanto, é uma importante condutora da inovação (SLATER; NARVER, 1995) e da habilidade de aprender sobre e de efetivamente explorar as novas oportunidades de mercado (SHANE; VENKATARAMAN, 2000). Hilt, Ireland, Camp and Sexton (2001) associam a orientação ao empreendedorismo como uma habilidade em fazer a incerteza do ambiente empresarial trabalhar de forma positiva para a empresa. 
Sob a ótica da abordagem baseada em recursos da empresa, empresas com forte orientação ao empreendedorismo são capazes de conectar um conjunto de capacidades e recursos através de um processo de aprendizagem, possibilitando a identificação de necessidades latentes dos clientes, além de promover o vínculo de novas tecnologias a serviço dessas necessidades (OZSOMER; CALANTONE; DI BENEDETTO, 1997). Com isto, é possível à empresa com alto nível de orientação ao empreendedorismo gerar inovações valiosas, únicas e difíceis de serem imitadas (DAY, 1994; HULT; KETCHEN 2001; MATSUNO; MENTZER,; OZSOMER, 2002), ou seja, a inovação radical.

Nesse contexto, a inovação radical refere-se a mudanças tecnológicas significativas não-lineares que representam um salto na performance e torna a antiga tecnologia obsoleta (MCKEE, 1992). A inovação radical traz novas plataformas de inovação, enquanto que a inovação incremental melhora e refina as plataformas existentes. Em geral, as inovações radicais trazem maiores benefícios à empresa do que as inovações incrementais, apesar das últimas ocorrerem com maior frequência (CHANDY; TELLIS, 2000). Produtos inteiramente novos trazem maior valor do que extensões de linha (CHANEY; DEVINNEY; WINER, 1991) e tecnologias inteiramente novas são mais rentáveis do que melhorias incrementais (SORESCU; CHANDY; PRAHBU 2003).

Dessa forma, a aplicação efetiva da aprendizagem promovida pela orientação ao empreendedorismo é considerada como um antecedente da geração de inovação radical e, finalmente, uma possível vantagem competitiva (CALANTONE; DI BENEDETTO; BHOOVARAGHAVAN, 1994). Assim, propõe-se que:

\section{H1: orientação ao empreendedorismo impacta positivamente a inovação radical.}

A geração de informação de mercado, no escopo da orientação para o mercado, e a orientação ao empreendedorismo, ainda que se sobreponham, têm domínios distintos (SLATER; NARVER, 1995). Ambas propagam comportamentos que facilitam o processamento e aprendizagem das informações do mercado (COVIN; MILES, 1999; SENGE 1990). As dimensões da orientação ao empreendedorismo - propensão ao risco, à pró-atividade e à inovação, assim como o processo de geração de informação de mercado, são baseadas na capacidade das empresas de rever o ambiente de mercado de forma eficaz (BAKER; SINKULA, 2002; SLATER; NARVER, 1995).

Huber (1996, p.822) postula que "uma organização aprende quando, através do seu processamento de informações, ela aumenta a probabilidade de que suas ações futuras promovam um acréscimo de performance". Com este intuito, o processo de geração de conhecimento na organização envolve a aquisição, a distribuição e a interpretação da informação, normalmente de forma interpessoal e social (HUBER, 1991), alimentando e utilizando uma assim chamada memória organizacional (WALSH; UNGSON, 1991). A geração de informação de mercado é considerada o ponto de partida do processo de aprendizagem organizacional (GARVIN, 1993).

Morgan e Turnell (2003) referem-se especificamente à geração de informação de mercado como a aprendizagem organizacional baseada no mercado. Segundo os autores tal tipo de aprendizagem envolve “... o desenvolvimento de conhecimento sobre concorrentes e mercados e as suas interações com esses mercados, o qual pode ser desenvolvido e armazenado em rotinas comportamentais, e que são geradas através de vários processos de informação e melhoradas através de valores distintivos organizacionais" (p. 256). A aprendizagem organizacional baseada no mercado caracteriza-se pelos seguintes aspectos: é uma competência central focada no ambiente externo da organização; gera vantagem competitiva fundamentada no mercado; estimula uma qualidade diferenciadora da organização através da constante monitorização da atuação da concorrência; e, consolida a correta interpretação de dados disponíveis para apoio à tomada de decisão/ação organizacional. De um modo geral, a perspectiva externa da aprendizagem organizacional baseada no mercado torna os processos mais complexos quando comparados com contextos de aquisição e de processamento de informação interna.

Huber (1996) sintetiza o conjunto de meios de aquisição de informações, afirmando que a organização aprende através das suas próprias experiências, através da inspeção e interação com o seu ambiente e, finalmente, pela compra propriamente dita da informação, seja pela formação de uma aliança com outra organização que detenha o conhecimento, seja pela contratação de pessoal com domínio da informação desejada.

Considerando que as empresas orientadas para o mercado são as mais capacitadas para identificar e atender às necessidades latentes dos consumidores, as mesmas podem ser tomadas como aquelas com maior probabilidade de serem as primeiras do mercado a lançar novas gerações de produtos e serviços existentes (DAY, 1994; BAKER; SINKULA, 1999; GATIGNON; XUEREB, 1997; KOHLI; JAWORSKI, 1990; NARVER; SLATER, 1990). Contudo, a inovação exitosa, conforme supracitado, requer o desenvolvimento de habilidades e recursos de forma singular (BARNEY, 1991; TEECE; PISANO; SHUEN, 1997), destacando-se o conhecimento como o recurso estrategicamente mais importante (DAY, 1994; HAMEL; PRAHALAD, 1994). Em geral, a criação de conhecimento procede, originalmente, do processo de geração de informação do mercado (ZAHRA; IRELAND; HITT, 2000). 
Essencialmente, na literatura específica sobre inovação, a oferta de tecnologia e a demanda de mercado são identificadas como as duas maiores fontes para idéias de inovação radical (LI; CALANTONE, 1998). De fato, Atuahene-Gima e Ko (2001) postulam que o desenvolvimento de inovação radical requer um maior conhecimento sobre o mercado, bem como o conhecimento sobre o estado-de-arte da tecnologia. Desta forma, sugere-se que:

\section{H2: A geração de informação de mercado impacta positivamente a inovação radical.}

O ponto de vista da teoria de redes sociais concentra-se na natureza de relacionamentos entre atores de uma rede social e os efeitos que essas relações trazem no compartilhamento de informações (KOKA; PRESCOTT, 2002). Nesta linha, situa-se o conceito de capital social que representa os valores embutidos nos relacionamentos entre pessoas imanentes das conexões sociais (CAO; MARUPING; TAKEUCHI, 2006), podendo ser interno ou externo à empresa. Dentro da empresa, a disseminação da comunicação intra e interdepartamental é facilitada pelo capital social interno, sendo bastante similar aos conceitos de disseminação da informação (JAWORSKI; KOHLI, 1993) e de coordenação interfuncional (NARVER; SLATER, 1990) subconstrutos estabelecidos dentro do construto de orientação para o mercado. O presente artigo, conforme supracitado, enfatiza o capital social externo que engloba estudos anteriores focados nas conexões sociais e relacionamentos pessoais de gestores com gestores de outras empresas (e.g., PENG; LOU, 2000), entre funcionários e clientes (e.g., LIAN; LAING 2007) e entre funcionários de diferentes empresas (e.g., HANSEN, 1999).

Esses relacionamentos de redes sociais são condutos de informações e fontes chaves de aprendizagem, isto é, as redes proporcionam acesso ao conhecimento que possibilita adaptação ao ambiente e identificação de oportunidades (BURT, 1992; KOKA; PRESCOTT, 2002; NAPHIET; GHOSHAL, 1998; YLI-RENKO; AUTIO; SAPIENZA, 2001). Dessa forma, o capital social externo é um meio que fornece informação e ideias à organização de uma diversidade de fontes externas, auxiliando o processo de tomada de decisão, identificando mudanças de demanda potenciais, melhoramentos tecnológicos e novas oportunidades de negócio (CAO; MARUPING; TAKEUCHI, 2006; COLLINS; CLARK, 2003; HANSEN, 1999).

No escopo do processo de aprendizagem organizacional, Coleman (1988) defende que o capital social externo impulsiona a produtividade das empresas, do seu capital humano e financeiro, que podem levar à vantagem competitiva. Florin, Lubatkin, e Schulze (2003) sugerem que a explicação sobre o capital humano se baseia nas qualificações das equipes, enquanto que a definição para o capital social externo é baseada nas credenciais da equipe quanto aos seus contatos sociais. Já Stam e Elfring (2008) afirma que o capital social externo facilita uma orientação ao empreendedorismo através da potencialização deste recurso com aspectos oriundos de outros domínios sociais. Neste sentido, Baker, Grinstein e Harmancioglu (2009) consideram que o capital social externo influencia os recursos humanos e financeiros e, desta maneira, alavanca aspectos da cultura organizacional que conduzem à inovação. Neste raciocínio, o capital social externo poderia ser visto como o catalisador de um "ciclo virtuoso" na relação entre a orientação ao empreendedorismo e a inovação, capaz de facilitar o desenvolvimento de aptidões únicas, além de permitir a sustentação de inovações de mais alto nível (HARRISON; BOYLE, 2006; STAM; ELFRING, 2008). Assim, considera-se que:

\section{H3: O capital social externo modera positivamente a relação entre a orientação ao empreendedorismo e a inovação radical.}

Por outro lado, o capital social externo pode fornecer informações importantes sobre os clientes, trazendo interpretações alternativas sobre os consumidores, concorrentes, tecnologias e outros elementos do ambiente do mercado da empresa (BAKER; GRINSTEIN; HARMANCIOGLU, 2009). Essas perspectivas do exterior podem agregar maior valor que a entrada bruta de dados que traz as informações do mercado e suas atividades de compra. É esta função interpretativa dentro das redes externas que pode servir para fornecer percepções de oportunidades com maior ou menor potencial para a empresa, reduzindo as incertezas a respeito do mercado, fortalecendo o impacto positivo da geração de informação do mercado na inovação radical. Com isso, postula-se que:

\section{H4: O capital social externo modera positivamente a relação entre a geração de informação de mercado e a inovação radical.}

A aglutinação das hipóteses supracitadas retrata a definição do modelo teórico testado. A Figura 1 expõe graficamente o modelo e as relações hipotetizadas. 


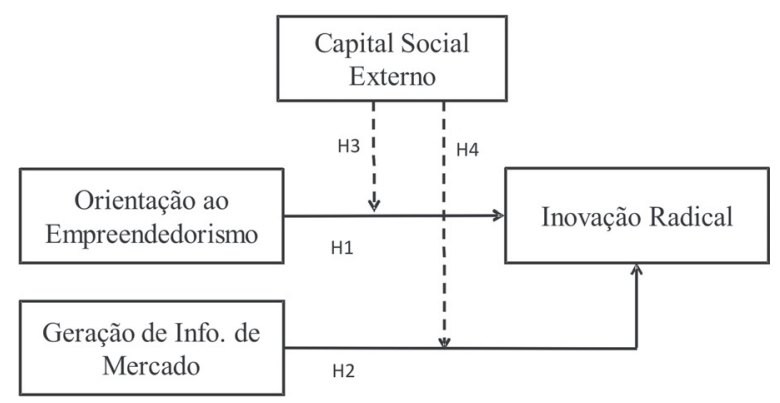

Figura 1 - Modelo Teórico da Relação entre os Construtos Fonte: elaborado pelos autores.

Os procedimentos metodológicos aplicados para a avaliação do modelo no contexto brasileiro são descritos a seguir.

\section{Método}

O método aplicado na realização desta pesquisa, dada a sua natureza descritiva, foi o levantamento do tipo survey cross-sectional, seguindo recomendações de Churchill (1999). A técnica utilizada para a avaliação do modelo proposto foi a análise fatorial confirmatória, através do uso de modelagem de equações estruturais (HOYLE, 1995; HAIR et al., 1998). A modelagem de equações estruturais é usualmente empregada em estudos de investigação de relações entre construtos tendo como base dados oriundos de levantamentos do tipo corte transversal. Através desta técnica, o pesquisador pode investigar modelos complexos caracterizados por uma série de equações descritivas das inter-relações entre diversas variáveis endógenas e exógenas (STEENKAMP; BAUMGARTNER, 2000).

Para Hair et al. (1998), a técnica de modelagem de equações estruturais é particularmente útil para verificar modelos teóricos mais complexos, nos quais variáveis dependentes se tornam independentes em relações de dependência subseqüentes. Essencialmente, a modelagem de equações estruturais é uma abordagem estatística abrangente que permite o teste de hipóteses sobre as relações entre variáveis observáveis e latentes (HOYLE, 1995).

A existência da direção e significância do efeito moderador do capital social externo foi feita através do teste qui-quadrado, seguindo recomendações de Sauer e Dick (1993) e Hoyle (1995) para a análise multigrupo em modelagem de equações estruturais, considerando os grupos extremos de conhecimento - alto e baixo. Para tanto, a amostra foi dividida em três grupos: empresas com baixo, médio e alto capital social. Considerando o modelo teórico, as análises foram delineadas para testar apenas a invariância dos parâmetros estruturais (causais), respeitando o procedimento adotado por Laroche, Bergeron e Goutaland (2003). Basicamente, esse procedimento consiste na análise da significância do qui-quadrado na comparação de dois modelos, sendo um com a estimação livre de parâmetros para a relação de impacto em análise e outro com a fixação dos parâmetros para essa mesma relação. A análise multigrupo foi realizada com a utilização do módulo correspondente no software estatístico AMOS ${ }^{\circledR}$.

Os detalhes quanto à elaboração dos instrumentos de coleta aplicados, bem como quanto ao processo de coleta de dados e das análises a realizadas, são apresentados a seguir.

\subsection{Elaboração do Instrumento de Coleta}

A validação do modelo teórico proposto exigiu a elaboração de instrumento de coleta para a mensuração dos seguintes construtos: orientação ao empreendedorismo, geração de informação de mercado, capital social externo e inovação radical.

Para operacionalização destes construtos foram utilizadas escalas já desenvolvidas, particularmente aquelas aplicadas no estudo de Baker, Grinstein e Harmancioglu (2009). Originalmente em inglês, todas as escalas foram convertidas para a língua portuguesa por meio da técnica de tradução reversa (DILLON; MADDEN; FIRTLE, 1994) e utilizaram uma notação de 5 pontos, do padrão Likert, a exceção do construto de geração de informação de mercado, já amplamente utilizado e validado no ambiente brasileiro.

Sinteticamente, o construto de capital social externo baseou-se na escala desenvolvida por Baker, Grinstein e Harmancioglu (2009), a qual considera o grau com que os empregados da empresas pesquisadas desenvolvem redes de comunicação externas para ter acesso a novas idéias, resolver problemas, buscar diferentes pontos de vista, explorar oportunidades e dar sentido a novas informações sobre o seu negócio. O construto de orientação ao empreendedorismo, por sua vez, foi mensurado a partir da escala proposta por Baker e Sinkula (2007), adaptada de Naman e Slevin (1993), englobando a propensão ao risco, à pro-atividade e à inovação. Para a mensuração da geração de informações de mercado foram considerados os itens correspondentes da escala MARKOR (KOHLI; JAWORSKI; KUMAR, 1993). Finalmente, a inovação radical foi operacionalizada com uma medida inicialmente proposta por Chandy e Tellis (1998), baseada na opinião dos executivos quando ao tipo de inovação priorizada em cada organização, envolvendo a freqüência com que novos produtos ou serviços são introduzidos no mercado, tornando obsoletos àqueles já existentes, promovendo mudanças fundamentais nos produtos principais e representando novas maneiras de satisfazer as necessidades dos consumidores. 
A validação de conteúdo do instrumento de coleta foi efetuada através do método de juízes, acadêmicos e práticos (CHURCHILL, 1999). Os juízes foram dois acadêmicos com experiência na aplicação dos conceitos da área de Marketing. Após essa etapa, para avaliação dos termos empregados, nos setores econômicos em estudo, o questionário foi apresentado a quatro executivos, escolhidos por julgamento do pesquisador, objetivando aproximar os termos empregados no instrumento de coleta a uma linguagem mais prática e mais específica dos setores em estudo. Complementarmente, o instrumento de coleta de dados foi pré-testado em um conjunto de empresas da população alvo, representando diferentes portes e localizações geográficas, com o objetivo de identificar e eliminar problemas potenciais de entendimento e preenchimento.

\subsection{Coleta de Dados}

A forma de coleta adotada foi o de questionário via correio. A população alvo do estudo incluiu empresas brasileiras de setores econômicos industriais e de serviços com mais de 250 funcionários, tendo sido enviados cerca de 3.000 questionários para empresas selecionadas da população, com base em cadastro adquirido junto à Dun \& Bradstreet do Brasil. Obteve-se um retorno de questionários válidos em torno de $11,4 \%$, perfazendo uma amostra final de 343 casos.

O destino do questionário foi a alta administração das empresas envolvidas, sendo encaminhado para o executivo de contato de cada empresa cujo nome constava do referido cadastro. Este procedimento partiu da premissa que há forte correlação entre as informações prestadas por informantes-chave (respondentes únicos) e por respondentes múltiplos (JAWORSKI; KOHLI, 1993; HOMBURG; WORKMAN; KROHMER, 1999).

Os questionários recebidos foram digitados e processados no software estatístico SPSS. Dados os métodos de coleta utilizados, foi realizada uma análise do viés dos não-respondentes através da comparação entre as características das empresas respondentes e não respondentes (dados relativos ao número de funcionários, localização geográfica e classificação pelo código de atividade), além do teste de comparação entre ondas (1a onda após o envio do questionário e $2 \mathrm{a}$ onda após a pós-notificação), sem a constatação de discrepância entre as ondas. A Tabela 1 apresenta uma síntese das características da população alvo e também da amostra utilizada no estudo, particularmente os dados de número de funcionários, ano de estabelecimento e setor de atividade (baseado no código SIC - Standard Industrial Classification).

Tabela 3 - Características da População / Amostra

\begin{tabular}{|c|c|c|c|}
\hline & & População & Amostra \\
\hline \multirow{4}{*}{$\begin{array}{l}\text { Número de } \\
\text { Funcionários }\end{array}$} & $250-400$ & $29,6 \%$ & $33,0 \%$ \\
\hline & $401-800$ & $27,1 \%$ & $28,5 \%$ \\
\hline & $801-1200$ & $15,1 \%$ & $10,2 \%$ \\
\hline & Acima de 1200 & $28,3 \%$ & $28,3 \%$ \\
\hline \multirow{4}{*}{ Fundação } & Até 1960 & $25,6 \%$ & $28,3 \%$ \\
\hline & $1961-1980$ & $33,2 \%$ & $34,3 \%$ \\
\hline & $1981-2000$ & $37,6 \%$ & $32,1 \%$ \\
\hline & Após 2000 & $3,5 \%$ & $5,3 \%$ \\
\hline \multirow{10}{*}{$\begin{array}{l}\text { Setor de } \\
\text { Atividade } \\
\text { (SIC) }\end{array}$} & Agricultura, Silvicultura, Pesca & $1,9 \%$ & $2,7 \%$ \\
\hline & Construção & $3,1 \%$ & $2,9 \%$ \\
\hline & Finanças, Seguros, Imóveis & $3,3 \%$ & $2,9 \%$ \\
\hline & Manufatura & $59,7 \%$ & $61,1 \%$ \\
\hline & Mineração & $0,9 \%$ & $1,9 \%$ \\
\hline & Administração Pública & $0,9 \%$ & $0,0 \%$ \\
\hline & Comércio Varejista & $4,3 \%$ & $3,2 \%$ \\
\hline & Serviços & $12,9 \%$ & $11,5 \%$ \\
\hline & $\begin{array}{l}\text { Transporte, Comunicação, } \\
\text { Elétrica e Gás }\end{array}$ & $8,1 \%$ & $8,8 \%$ \\
\hline & Comércio atacadista & $4,8 \%$ & $5,1 \%$ \\
\hline
\end{tabular}

\section{Análise dos Resultados}

Neste estudo, a avaliação das relações estruturais seguiu os passos recomendados por Hair et. al. (1998), utilizando-se o software estatístico AMOS ${ }^{\circledR}$. O exame das propriedades relativas à validade dos construtos, quais sejam, a unidimensionalidade, a confiabilidade, a validade convergente e a validade discriminante, seguiu recomendações de Garver e Mentzer (1999). Todos os construtos envolvidos no estudo demonstraram validade de construto. A Tabela 2 apresenta a média, o desvio padrão, a confiabilidade de construto, a correlação e o quadrado da variância extraída referentes aos construtos em análise.

Tabela 2 - Parâmetros Estimados do Modelo

\begin{tabular}{|c|cccccc|}
\hline & Média & DP & CC & OE & GIM & IR \\
\hline $\begin{array}{c}\text { Orientação ao } \\
\text { Empreendedorismo }\end{array}$ & 4,64 & 1,13 & 0,75 & $\mathbf{0 , 6 6}$ & & \\
\hline $\begin{array}{c}\text { Geração de Informação } \\
\text { de Mercado }\end{array}$ & 5,30 & 1,09 & 0,78 & 0,335 & $\mathbf{0 , 6 5}$ & \\
\hline Inovação Radical & 3,90 & 1,29 & 0,78 & 0,500 & 0,241 & $\mathbf{0 , 7 5}$ \\
\hline
\end{tabular}

Notas: Os valores apresentados em negrito na linha diagonal representam a raiz quadrada da variância extraída relativa a cada construto. 
Os indicadores de estimação utilizados para a validação do modelo foram selecionados do conjunto proposto por Hair et al. (1998). Os indicadores considerados têm sido frequentemente empregados em artigos que utilizam modelagem de equações estruturais. Destaca-se que existem várias medidas de ajustamento desenvolvidas, absolutas e comparativas, não existindo, portanto, um único coeficiente que resuma o ajustamento do modelo. Além disso, não existem parâmetros rígidos para essas medidas para aceitação ou rejeição de um modelo, dependendo de fatores como a complexidade do modelo proposto.

As medidas absolutas de ajustamento aplicadas, que determinam o grau em que o modelo geral prediz a matriz de covariância ou de correlação observada (HAIR et al., 1998), foram: qui-quadrado sobre graus de liberdade ( $\chi 2$ / GL), Índice de Adequação de Ajustamento (GFI) e Erro de Aproximação do Quadrado Médio da Raiz (RMSEA). Já as medidas comparativas de ajustamento, que comparam o modelo proposto ao modelo nulo (null model), foram: Índice de Adequação de Ajustamento Ajustado (AGFI), Índice de TuckerLewis (TLI) e Índice de Ajustamento Comparativo (CFI).

Na aplicação da técnica de modelagem de equações estruturais foram analisadas as medidas de ajustamento do modelo teórico, o nível de significância e a magnitude dos coeficientes de regressão estimados para as relações propostas pelas hipóteses do modelo. Para tanto, seguiu-se a recomendação explícita oferecida pela empresa SmallWaters Corp., produtora do software estatístico $\mathrm{AMOS}^{\circledR}$ utilizado na pesquisa. Dado o diagnóstico de não normalidade não substancial dos dados da amostra (skewness menor ou igual a dois e kurtosis menor ou igual a sete) (HOYLE, 1995) e do uso de variáveis categóricas do tipo Likert com cinco ou mais pontos, a empresa sugere que seja aplicada a abordagem maximum likelihood (ML), considerando a variável categórica como intervalar e aplicando o processo de estimação de bootstrapping para a correção dos erros padronizados.

O exame da significância e magnitude dos parâmetros estimados (cargas fatoriais padronizadas), por sua vez, permite a verificação das relações estabelecidas no modelo teórico. A identificação da significância de um coeficiente de regressão (parâmetro estimado) é realizada pela análise do seu respectivo $t$-value. Valores de $t$-values superiores a 1,96 definem um nível de significância de no mínimo 0,05 (GARVER; MENTZER, 1999). A constatação de um coeficiente de regressão significativo implica a consideração de que a relação entre as duas variáveis se comprova empiricamente (HAIR et al., 1998) e, no caso da avaliação positiva ou satisfatória das medidas de ajustamento, destaca a validade preditiva do modelo (GARVER; MENTZER, 1999).
Com o intuito de aprofundar os resultados identificados e, principalmente, testar a invariâncias das relações estruturais e do modelo testado como um todo, além da influência moderadora do capital social externo, verificou-se possíveis diferenças no comportamento dos parâmetros estimados entre empresas com baixo e alto nível de capital social externo. As comparações foram feitas através do teste qui-quadrado, tomando-se os grupos de empresas dois a dois, aplicando-se a análise multigrupo em modelagem de equações estruturais (SAUER; DICK, 1993; HOYLE, 1995).

A Tabela 3 relaciona os parâmetros estimados para as relações, bem como os parâmetros de ajustamento de cada um dos contextos analisados para o modelo teórico, além dos valores de qui-quadrado relativos à comparação entre as realidades das subamostras.

Tabela 3 - Parâmetros Estimados do Modelo

\begin{tabular}{|c|c|c|c|}
\hline & Geral & CSE baixo & CSE alto \\
\hline $\mathrm{H}_{1}-\mathrm{OE}>>\mathrm{IR}$ & $0,614^{* *}$ & $0,535^{* *}$ & $0,689^{* *}$ \\
\hline $\mathrm{H}_{2}-\mathrm{GIM} \gg>\mathrm{IR}$ & 0,055 & $0,233^{*}$ & $-0,143$ \\
\hline \multicolumn{4}{|c|}{ Medidas de Ajustamento } \\
\hline Q.Q. / GL & 2,117 & 1,385 & 1,563 \\
\hline RMSEA & 0,057 & 0,05 & 0,06 \\
\hline GFI & 0,949 & 0,93 & 0,918 \\
\hline AGFI & 0,923 & 0,897 & 0,875 \\
\hline TLI & 0,932 & 0,941 & 0,924 \\
\hline CFI & 0,948 & 0,954 & 0,941 \\
\hline \multicolumn{4}{|c|}{ Comparação - Dif. de Qui Quadrado } \\
\hline $\mathrm{H} 3-\mathrm{OE}>>\mathrm{IR}$ & & 0,009 & \\
\hline H4 - GMI > IR & & $6,233^{*}$ & \\
\hline
\end{tabular}

Fonte: Coleta de dados

Notas: Os valores apresentados nas primeiras linhas representam a carga fatorial padronizada registrada para cada relação. Os valores nas linhas intermediárias representam os índices de ajustamento de cada modelo. Os valores apresentados nas últimas linhas representam o valor do qui-quadrado na comparação dos modelos. OE orientação ao empreendedorismo; GIM - geração de informação de mercado; CSE - capital social externo; IR - inovação radical. *** $\mathrm{p}<0,01{ }^{*} \mathrm{p}<0,05$

Em termos gerais, o modelo foi considerado ajustado para a realidade brasileira, ainda que no caso específico das subamostras tenha sido registrado um indicador levemente abaixo do padrão ideal de ajustamento AGFI igual a 0,897 e 0,875, respectivamente (HAIR et. Al, 1998).

Na Tabela 1, pode-se verificar que a hipótese H1 foi confirmada nas duas subamostras, demonstrando a fortaleza do impacto que a orientação ao empreendedorismo exerce sobre a inovação radical $(\beta=0,614, p<0,01)$. Já a hipótese $\mathrm{H} 2$ foi confirmada parcialmente, uma vez que para o conjunto de empresas brasileiras com baixo nível de capital social externo o impacto da geração de informação de mercado se mostrou significativa $(\beta=0,233, p<0,05)$, embora a realidade brasileira como 
um todo apresente uma carga não significativa de efeito direto nesta relação $(\beta=0,055, p>0,05)$, aparentemente influenciada pela condição avaliada nas suas empresas com alto capital social externo $(\beta=-0,143, p>0,05)$.

Note-se que os resultados registrados na Tabela 1 oferecem, ainda, rejeição à hipótese relativa à ação de moderação do capital social externo no efeito da orientação ao empreendedorismo sobre a inovação radical $\left(\mathrm{H}_{3}\right)$. Embora essa relação tenha apresentado diferença em sua magnitude entre os grupos de alto e baixo nível de capital social, esta diferença não é significativa $(\chi 2=0,009, p>0,05)$. Registra-se, contudo, que o impacto da orientação ao empreendedorismo sobre a inovação radical foi maior no caso de níveis mais elevados de capital social externo. Em outras palavras, o capital social externo não demonstrou interferência significativa sobre a relação em questão, apesar de ter sido constatado um leve incremento na importância da relação. Numa situação inversa, o efeito moderador da geração de informação sobre a inovação radical $\left(\mathrm{H}_{4}\right)$ se mostrou significativo $(\chi 2=6,233, \mathrm{p}<0,01)$, porém com influência negativa no impacto da relação, registrando resultado inverso ao proposta na hipótese $\mathrm{H}_{4}$.

As considerações finais quanto a estes resultados são apresentadas no item a seguir.

\section{Conclusões e Implicações Acadêmicas e Gerenciais}

Este trabalho relacionou as proposições desenvolvidas por Baker, Grinstein e Harmancioglu (2009), relativas ao papel moderador do capital social externo no impacto dos antecedentes da inovação radical, particularmente a orientação ao empreendedorismo e a geração de informação de mercado.

O modelo teórico, proposto e verificado, apresentou uma influência direta, forte e positiva da orientação ao empreendedorismo sobre o desenvolvimento da inovação radical, dentro da realidade brasileira, demonstrando que a propensão ao risco, à pro-atividade e à inovação, estimulam a busca pela introdução no mercado de novos produtos ou serviços que tornem obsoletos aqueles já existentes, promovendo mudanças fundamentais nos produtos principais e representando novas maneiras de satisfazer as necessidade dos consumidores. Estes resultados estiveram coerentes com resultados anteriores dispostos na literatura da área (OZSOMER; CALANTONE; DI BENEDETTO, 1997; DAY 1994; HULT; KETCHEN, 2001; MATSUNO; MENTZER; OZSOMER, 2002).

No que se refere à geração de conhecimento a partir do mercado, as empresas brasileiras não conseguem potencializar o uso desse conhecimento para inovar radicalmente, contrariando a hipótese anteriormente definida. Aparentemente, o fortalecimento de redes de comunicação externas para ter acesso a novas ideias, resolver problemas, buscar diferentes pontos de vista, explorar oportunidades e dar sentido a novas informações sobre o seu negócio (SLATER; NARVER, 1995), não é uma prática de empresas brasileiras.

Essencialmente, o que se desenha nos resultados encontrados é uma situação em que a intensificação do capital social externo não registra impacto significativo no caráter empreendedor das empresas quando do desenvolvimento de inovação radical. Ao contrário, surpreendentemente diminuiu significativamente a força ou participação das informações advindas do mercado neste processo de desenvolvimento. Este achado pode estar relacionado à natureza da estrutura da rede entre as empresas brasileiras, estrutura na qual as empresas tendem à efetivação de conglomerados e ao controle sobre o mercado / ambiente (AMAN; BAUER, 2008). Nesse contexto, o capital social externo seria mais aplicado na tentativa de estabelecimento de acordos de domínio de mercados, ao invés da busca por potencialização e melhor entendimento das informações extraídas do mercado.

Em termos gerais, o bojo de empresas brasileiras atua focando intensamente na sua orientação empreendedora para a efetivação de inovações radicais, ignorando neste processo a possibilidade de utilização das informações que por ventura tenham sido obtidas do seu mercado. Basicamente, as empresas brasileiras não conhecem ou não fazem uso das decorrências do seu capital social externo, ao menos quando se trata da busca pela inovação radical.

Por fim, numa visão de rede nomológica, a pesquisa apresentou e testou uma estrutura de relações entre a orientação ao empreendedorismo, geração de informação de mercado e inovação radical, além de descrever o efeito moderador do capital social externo nestas relações. Neste sentido, o presente estudo contribui com o esforço acadêmico na área de marketing para a definição dos caminhos pelos quais os recursos e habilidades de marketing influenciam a performance das organizações, especialmente o desenvolvimento de inovação radical.

\section{Limitações da Pesquisa e Sugestões para Estudos Futuros}

Apesar do rigor do método aplicado nessa pesquisa, algumas situações vivenciadas durante o desenvolvimento do trabalho sugerem certas limitações, e a superação de cada uma dessas limitações resultará em recomendações para pesquisas futuras. 
“(...) As associações em um modelo de equações estruturais são evidências necessárias, mas não suficientes de relações causais" (HOYLE, 1995, p. 175). Assim sendo, os resultados confirmatórios aqui apresentados e discutidos, concernentes às relações entre os construtos do modelo proposto, devem ser considerados tão-somente como evidências de relação causal entre os referidos construtos.

A percepção de um único respondente por unidade amostral pode distorcer as verdadeiras práticas adotadas na organização. Desta forma, seria importante que estudos futuros considerassem outras possibilidades, tais como respondentes múltiplos, respondentes de outros escalões da organização ou ainda respondentes externos a organização.

Finalmente, apesar da preocupação com a análise do viés dos não respondentes, salienta-se que essa pesquisa utilizou amostras não probabilísticas de retorno de correio. Seria interessante que trabalhos futuros utilizassem mais de um método de coleta de dados, observando a aleatoriedade na seleção das unidades amostrais.

\section{Referências Bibliográficas}

AMANN, Edmund; BAER, Werner. Neo-liberalism and market concentration in Brazil: The emergence of a contradiction? The Quarterly Review of Economics and Finance, v. 48, p. 252-262, 2008.

ATUAHENE-GIMA, Kwaku. Market orientation and innovation. Journal of Business Research, v. 35, n. 2, p. 93-103, Feb. 1996.

BAKER, William E.; SINKULA, James M. Does Market Orientation Facilitate Balanced Innovation Programs? An Organizational Learning Perspective. The Journal of Product Innovation Management, vol. 24, n. 4, p. 316-34, 2007.

BAKER, William E.; SINKULA, James M. Learning Orientation, Market Orientation, and Innovation: Integrating and Extending Models of Organizational performance. Journal of Market-Focused Management, v. 4, n. 4, p. 295-308, Dec. 1999.

BAKER, William E.; SINKULA, James M. Market Orientation and The New Product Paradox. The Journal of Product Innovation Management, v. 22, p. 483-502, 2005.

BAKER, William E.; SINKULA, James M. Market Orientation, Learning Orientation and Product Innovation: Delving into the Organization's Black Box. Journal of Market - Focused Management, vol. 5, n. 1, p. 5-23., 2002.
BAKER, William; GRINSTEIN, AMIR; HARMANCIOGLU, Nukhet. External Social Capital: A Risk Mitigating Resource Of The Innovative Firm. Working paper, 2009.

BARNEY, J. B. Firm Resources and Sustained Competitive Advantage. Journal of Management, v.17, n. 1, p. 99-120, 1991.

BURT, Ronald. Structural Holes: The Social Structure of Competition. Cambridge, MA: Harvard University Press, 1992.

CALANTONE, Roger J. and DI BENEDETTO, C.; BHOOVARAGHAVAN, Sriraman. Examining the Relationship between Degree of Innovation and New Product Success. Journal of Business Research, vol. 30, p. 143-148, 1994.

CAO, Qing; MARUPING, Likoebe; TAKEUCHI, Riki. Disentangling the Effects of CEO Turnover and Succession on Organizational Capabilities: A Social Network Perspective. Organization Science, vol. 17, n. 5, p. 563-76, 2006.

CASEY, Nicholas; ORWALL, Bruce. Running Underground to Sharpen Nike's Edge, CEO Taps 'Influencers'. Wall Street Journal, October 24, A1, 2007.

CHANDY, Rajesh K. TELLIS, Gerard J. Organizing for Radical Product Innovation: The Overlooked Role of Willingness to Cannibalize. Journal of Marketing Research, vol. 35, n. 4, p. 474-87, 1998.

CHANDY, Rajesh K; TELLIS, Gerard J. The incumbent's curse? Incumbency, size, and radical product innovation. Journal of Marketing, vol. 64, n. 3, p. 1-17, 2000.

CHANEY, P. K.; DEVINNEY, T. M.; WINER, R. S. The impact of new product introductions on the market value of firms. Journal Business, v. 64, n. 4, p. 573-610, 1991.

CHURCHILL, Gilbert A, Jr. Marketing Research: Methodological Foundations. Orlando: The Dryden Press, 1999.

COLEMAN, James S. Social Capital in the Creation of Human Capital. American Journal of Sociology, vol. 94 (Suppl.), p. S95-S120, 1998.

COLLINS, Christopher; CLARK, Kevin. Strategic Human Resource Practices, Top Management Team Social Networks, and Firm Performance: The Role of Human Resource Practices in Creating Organizational Competitive Advantage. Academy of Management Journal, vol. 46, n. 6, p. 740-51, 2003. 
CONANT, J. S.; MOKWA, M. P.; VARADARAJAN, P. R. Strategic types, distinctive marketing competencies and organisational performance: a multiple measuresbased study. Strategic Management Journal, Vol. 11, pp. 365-383, 1990.

COVIN, J.; MILES, M. Corporate entrepreneurship and the pursuit of competitive advantage. Entrepreneurship Theory and Practice, v. 23, n. 3, p. 47-64, 1999.

COVIN, J.; SLEVIN, D. A Conceptual Model of Entrepreneurship As Firm Behavior. Entrepreneurship Theory and Practice, v. 16, n. 1, p. 7-26, 1991.

DAY, George S. The Capabilities of Market-Driven Organizations. Journal of Marketing, v. 58, n. 4, p 37-52, October, 1994.

FLORIN, Juan; LUBATKIN, Michael; SCHULZE, William. A Social Capital Model of High-Growth Ventures. Academy of Management Journal, vol. 46, n. 3, p. 374-84, 2003.

FORNELL, Claes; LARCKER, David F. Evaluating Structural Equation Models with Unobservable Variables and Measurement Error. Journal of Marketing Research, vol. 18, n. 1, p. 39-50, 1981

GARVER, M. S.; MENTZER, J. T. Logistics research methods: employing structural equation modeling to test for construct validity. Journal of Business Logistics, v. 20, n. 1, p. 33-57, 1999.

GARVER, M. S.; MENTZER, J. T. Logistics research methods: employing structural equation modeling to test for construct validity. Journal of Business Logistics, v. 20, n. 1, p. 33-57, 1999.

GARVIN, David A. Building a Learning Organization. Harvard Business Review, v. 71, n. 4, p. 78-91, JulyAug. 1993.

GIELENS, Katrijn; STEENKAMP, Jan-Benedict E.M. Drivers of Consumer Acceptance of New Packaged Goods: An Investigation Across Products and Countries. International Journal of Research in Marketing, vol. 24, p. 97-111, 2007.

GRAY, Brendan J.; HOOLEY, Graham. Market orientation and service firm performance: a research agenda. European Journal of Marketing, v. 36, n. 9/10, p. 980 - 988, 2002.

GRAYSON, Kent. Friendship versus Business in Marketing Relationships. Journal of Marketing, vol. 71, n. 4, p. 121-39, 2007.
HAIR, Joseph F., Jr.; ANDERSON, Rolph E.; TATHAM, Ronald L.; BLACK, William C. Multivariate Data Analysis. 5a Ed. New Jersey: Prentice Hall, 1998.

HAMEL, Gary; PRAHALAD, C. K. Competing for the Future. Harvard Business Review, vol. 72, n.4, p. 122-28, 1994.

HAN, Jin K.; KIM, Namwoon; SRIVASTAVA, Rajendra K. Market orientation and organizational performance: is innovation a missing link?. Journal of Marketing, v. 62, n. 4, p. 30-45, Oct. 1998.

HANSEN, M.T. “The search-transfer problem: The role of weak ties in sharing knowledge across organization subunits. Administrative Science Quarterly, vol. 44, p.82-112, 1999.

HANSEN, Morten. The Search Transfer Problem: The Role of Weak Ties in Sharing Knowledge across Organization Subunits. Administrative Science Quarterly, vol. 44, p. 82-111, 1999.

HARRISON, Jeannette; BOYLE, Emily. Falling into Capability Learning Traps: The Role of the Firm's Predominant Managerial Mental Models. Management Decision, vol. 44, n. 1, p. 31-43, 2006.

HITT, Michael; IRELAND, Duane; CAMP, Michael; SEXTON, Donald. Introduction to the Special Issue Strategic Entrepreneurship: Entrepreneurial Strategies for Wealth Creation. Strategic Management Journal, vol. 22, p. 479-91, 2001.

HOMBURG, Christian; WORKMAN, John P.; KROHMER, Harley. Marketing's influence within the firm. Journal of Marketing, v. 63, n. 2, p. 1-17, Apr. 1999.

HOYLE, Rick H (editor). Structural Equation Modeling: Concepts, Issues, and Applications. London: SAGE, 1995.

HUBER, George P. Organizational learning: a guide for executives in technology-critical organizations. International Journal Technology Management, Special Issue on Unlearning and Learning for Technological Innovation, v. 11, n. 7/8, p. 821-832, 1996.

HUBER, George P. Organizational learning: the contributing process and the literatures. Organizational Science, v. 2, n.1, p. 88-115, 1991.

HULT, Tomas; KETCHEN, David. Does Market Orientation Matter? A Test of the Relationship between Positional Advantage and Performance. Strategic Management Journal, vol. 22, p. 899-906, 2001. 
JAWORSKI, Bernard J.; KOHLI, Ajay K. Market Orientation: Antecedents and Consequences. Journal of Marketing, v. 57, p. 53-71, July 1993.

KOHLI, Ajay K.; JAWORSKI, Bernard J. Market Orientation: The Construct, Research Propositions, and Managerial Implications. Journal of Marketing, v. 54, n. 2, p. 1-18, Apr. 1990.

KOHLI, Ajay K.; JAWORSKI, Bernard J. Market Orientation: The Construct, Research Propositions, and Managerial Implications. Journal of Marketing, v. 54, n. 2, p. 1-18, Apr. 1990.

KOHLI, Ajay K.; JAWORSKI, Bernard J.; KUMAR, Ajith. MARKOR: A measure of Market Orientation. Journal of Marketing Research, v. 30, n. 4, p. 467-477, Nov. 1993.

KOKA, Balaji, PRESCOTT, John. Strategic Alliances as Social Capital: A Multidimensional View. Strategic Management Journal, vol. 23, p. 795-816, 2002.

LAROCHE, Michel; BERGERON, Jasmin; GOUTALAND, Christine. How Intangibility Affects Perceived Risk: the moderating role of knowledge and involvement. The Journal of Services Marketing, v. 17, n. 2, p. 122-140, 2003.

LEVINTHAL, Daniel; MARCH, James. The Myopia of Learning. Strategic Management Journal, vol. 14, p. 95-112, 1993.

LI, Tiger; CALANTONE, Roger J. The Impact of Market Knowledge Competence on New Product Advantage: Conceptualization and Empirical Examination. Journal of Marketing, vol. 62, n. 4, p. 13-29, 1998.

LIAN, Paul; LAING, Angus. Relationship in the Purchasing of Business to Business Professional Services: The Role of Personal Relationships, Industrial Marketing Management, 36, 709-718, 2007.

LUMPKIN, G. T.; DESS, Gregory G. (Clarifying the Entrepreneurial Orientation Construct and Linking it to Performance. The Academy of Management Review, vol. 21, n.1, p. 135-72, 1996.

MATSUNO, Ken; MENTZER John; OZSOMER, Aysegul. The Effects of Entrepreneurial Proclivity and Market Orientation on Business Performance. Journal of Marketing, vol. 66, n. 3, p. 18-32, 2002.

MOORMAN, Christine. Organizational Market Information Processes: Cultural Antecedents and New Product Outcomes. Journal of Marketing Research, v. 32, n. 3, p. 318-335, Aug. 1995.
MORGAN, R.E., TURNELL, C.R. Market-based organisational learning and market performance gains. British Academy of Management, vol. , p.255-74, 2003.

NAHAPIET, Janine; GHOSHAL, Sumantra. Social Capital, Intellectual Capital, and the Organizational Advantage. The Academy of Management Review, vol. 23 , n. 2, p. 242-66, 1998.

NAMAN, J.; SLEVIN, D. Entrepreneurship and the concept of fit: a model and empirical tests. Strategic Management Journal, v. 14, n. 2, p. 137-154, 1993.

NARVER, John C.; SLATER, Stanley F. The effect of a market orientation on business profitability. Journal of Marketing, v. 54, n. 4, p. 20-35, 1990.

PENG, Mike; LOU, Yadong. Managerial Ties and Firm Performance in a Transition Economy: The Nature of a Micro-Macro Link. Academy of Management Journal, vol. 43, n. 3, p. 486-501, 2000.

PERRY-SMITH, Jill; SHALLEY, Christina. The Social Side of Creativity: A Static and Dynamic Social Network Perspective. The Academy of Management Review, vol. 28, n.1, p. 89-106, 2003.

RINDFLEISCH, Aric; MOORMAN, Christine. The Acquisition and Utilization of Information in New Product Alliances: A Strength-of-Ties Perspectives. Journal of Marketing, vol. 65, n. 2, p. 1-18, 2001.

ROWLEY, Tim; BEHRENS, Dean; KRACKHARDT, David. Redundant Governance Structures: An Analysis of Structural and Relational Embeddedness in the Steel and Semiconductor Industries. Strategic Management Journal, vol. 21, p. 369-86, 2000.

SAUER, Paul L.; DICK, Alan. Using Moderator Variables in Structural Equation Models. Advances in Consumer Research, v. 20, n.1, p. 636-640, 1993.

SENGE, Peter. The Fifth Discipline: The Art and Practice of the Learning Organization. New York: Doubleday Currency, 1990.

SHANE, Scott; VENKATARAMAN, S. The Promise of Entrepreneurship as a Field of Research, The Academy of Management Review, vol. 25, n. 1, p. 217-26, 2000.

SINKULA, James M.; BAKER, William E.; NOORDEWIER, Thomas. A Framework for Market-Based Organizational Learning: Linking Values, Knowledge, and Behavior. Journal of the Academy of Marketing Science, vol. 25, n. 4, p. 305-18, 1997. 
SLATER, Stanley F.; NARVER, John C. Market Orientation and the Learning Organization. Journal of Marketing, v. 59, n. 3, p. 63-74, 1995.

SORESCU, Alina B.; CHANDY, Rajesh K.; PRABHU Jaideep C. Sources and Financial Consequences of Radical Innovation: Insights from Pharmaceuticals. Journal of Marketing, vol. 67, n. 4, p. 82-102, 2003.

STAM, Wouter; ELFRING, Tom. Entrepreneurial Orientation and New Venture Performance: The Moderating Role of Intra- and Extraindustry Social Capital. Academy of Management Journal, vol. 51, n. 1, p. 97-111, 2008.

STEENKAMP, Jan-Benedict E. M.; BAUMGARTNER, Hans. On the use of structural equation models for Marketing modeling. International Journal of Research in Marketing, v. 17, n. 2/3, p. 195-202, Sep. 2000.

TEECE, David J.; PISANO, Gary; SHUEN, Amy. Dynamic Capabilities and Strategic Management. Strategic Management Journal, vol. 18, n. 7, p. 50933, 1997.
TYAGI, Rajeev. New Product Introductions and Failures Under Uncertainty. International Journal of Marketing, vol. 23, p. 199-213, 2006.

VIEIRA, Valter A. The Relationship Between Market Orientation and Business Performance: a Brazilian Meta-Analysis. EMA 2008.

WALSH, James P.; UNGSON, Gerard R. Organizational Memory. Academy of Management Review, v. 16, n. 1, p. 57-91, 1991.

YLI-RENKO, H.; AUTIO, E.; SAPIENZA, H.J. Social capital, knowledge acquisition, and knowledge exploitation in young technology-based firms. Strategic Management Journal, vol. 22, p. 587-613, 2001.

ZAHRA, S.A.; IRELAND, R.D.; HITT, M.A. International expansion by new venture firms: International diversity, mode of market entry, technological learning, and firm performance. Academy of Management Journal, vol. 43, n. 5, p. 925-950, 2000.

Recebido em: 06/07/2011. Aceito em: 05/09/2011. 